\title{
Chinese Grounded Theory Based on the Bibliometric Analysis of CSSCI Journals in Recent Ten Years
}

\author{
Sijia Chu ${ }^{1}$ and Wenxin Guo ${ }^{1, *}$ \\ ${ }^{1}$ School of Government, Beijing Normal University, Beijing 100875, China \\ *Corresponding author. Email:201821260028@mail.bnu.edu.cn
}

\begin{abstract}
Grounded theory, as a hot topic in management research, is widely used by scholars due to its scientific nature and objectivity. In this work, 466 Chinese kinds of literature published in CSSCI journals from 2011 to 2020 were selected, and CiteSpace V5.7 software was used to comprehensively describe the characteristics of published quantity, authors, issuing institutions, etc. Also, keywords and their clustering, emergent words, time plot, etc., were analyzed and interpreted. It is found that although grounded theory started late in China, it has been gradually rising and recognized by scholars. On the other hand, it has been widely studied in the fields of management, education, economics, sports, etc., and has a more in-depth study on influencing factors and theoretical construction. It is hoped that the localization research and application of grounded theory in the field of Chinese management will be further discussed.
\end{abstract}

Keywords: Grounded theory; China; CiteSpace; Core journal

\section{INTRODUCTION}

Scholars point out that the effective way to explore the analysis is not the traditional empirical method, but the qualitative analysis, which is the insight, interpretation and construction of the deep structure and mechanism [1]. Among many analytical methods, the coding method based on grounded theory is very popular and is an excellent exploration method [2]. The main purpose of grounded theory is to establish theory based on the empirical data, emphasizing the discovery of problems from documents and situations. Through the continuous enrichment of the original data and the analysis of complex data collection and literature review, the concept and construction theory were refined. It is considered to be the most objective and scientific qualitative research method.

Nowadays, there are three popular schools of grounded theory: classical grounded theory school, procedural grounded theory school and constructive grounded theory school. These three schools of thought intersect and differ from each other [3]. In 1967, Glaser et al. put forward the grounded theory for the first time, which has been widely applied in the fields of nursing, pedagogy, religion, management, etc. In the long-term development, three schools of thought have emerged. The classical grounded theory school, represented by Glaser et al., emphasizes the natural emergence from data to reduce human subjectivity as much as possible. The procedural grounded theory school, represented by Strauss et al., focuses on using presupposition to link the existing experience and hypothesis theory to explore and sort out the rules. The constructive grounded theory school, represented by
Charmaz, inherits and carries forward the former two university schools and integrates their advantages [4]. Academic journals are one of the main carriers of academic research results. Bibliometric analysis of journals with strong academic influence is conducive to accurately grasp the hot areas of academic research. By analyzing and studying the literature on grounded theory in CSSCI journals, the hot issues and development trend of grounded theory research in the field of management in China can be understood, thus providing reference for more researchers and the next step of research.

\section{RESEARCH METHODS AND DATA SOURCES}

Bibliometrics is a research method which takes academic literature as the research object, analyzes the law of literature from the quantitative perspective, and shows the hot spot and development trend of the discipline research. The common tools of bibliometrics are CiteSpace, BibExcel, Pajek, etc. Among them, CiteSpace software has become the mainstream research tool of domestic bibliometric research because of its easy to learn operation and good visualization effect.

In the advanced retrieval of CNKI, 466 Chinese academic journals were obtained by taking "subject $=$ grounded theory" and "literature source = CSSCI (precision)" as the retrieval conditions, and 2011-2020 as the publishing time condition to limit the literature retrieval time. 


\section{ANALYSIS OF RESEARCH CONTENT}

\subsection{Integral analysis}

According to the statistics, the number of the grounded theory published in China showed a continuous rising trend in the early stage, and it bloomed in all fields. It is not only applied in the field of management, but also widely used in pedagogy, medicine, psychology, news media, etc. Based on the statistical analysis of 466 authors and publishing institutions, it can be seen that the number of papers published by most scholars tends to be balanced, and the relationship and cooperation between most authors do not show a close relationship. In response to the author, scholars will form the cohesion of their departments, majors and disciplines, and guide them in the research direction. There are also many high-yield institutions, such as management schools and business schools, which are closely related to the application of grounded theory in the field of management, thus forming a strong professional advantage.

\subsection{Keywords knowledge graph}

Word frequency analysis is a bibliometric method to determine the research hotspots and development trends in a certain research field by using the frequency of keywords or subject words that can reveal or express the core content of the literature. Keywords are the author's highly summary and concise of the article, which is the essence of the literature, and also an important index for the bibliometric analysis. Therefore, keyword is set as the node type through CiteSpace V5.7 software in this work. A metrological visualization analysis was conducted on the literature of domestic core management journals from 2011 to 2020 . The keyword knowledge graph can be obtained, as shown in Fig. 1.

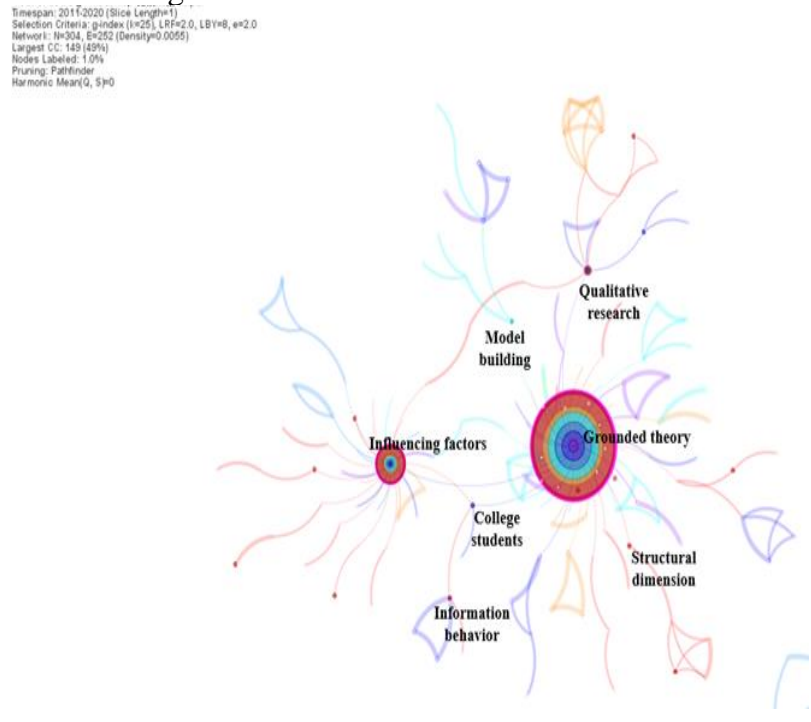

Figure 1. Keyword knowledge graph in grounded theory literature
Table 1 specifically reflects the high-frequency keywords of journal literature. The research content is carried out around the grounded theory, influencing factors, qualitative research and other keywords, and mainly focuses on theoretical construction, research methods and other perspectives.

Table 1. High-frequency keywords in grounded theory literature

\begin{tabular}{cccc}
\hline Keyword & Frequency & $\begin{array}{c}\text { Mediation } \\
\text { centrality }\end{array}$ & $\begin{array}{c}\text { First } \\
\text { time } \\
\text { year }\end{array}$ \\
\hline $\begin{array}{c}\text { Grounded } \\
\text { theory }\end{array}$ & 335 & 0.42 & 2011 \\
$\begin{array}{c}\text { Influencing } \\
\text { factors }\end{array}$ & 50 & 0.2 & 2011 \\
$\begin{array}{c}\text { Qualitative } \\
\text { research } \\
\text { Information } \\
\text { behavior } \\
\text { Model } \\
\text { building } \\
\text { College }\end{array}$ & 14 & 0.09 & 2011 \\
students & 5 & 0.02 & 2012 \\
$\begin{array}{c}\text { Structural } \\
\text { dimension }\end{array}$ & 5 & 0.03 & 2020 \\
\hline
\end{tabular}

\subsection{Clustering tag atlas}

In order to further explore the research hotspots in this field, this study used CiteSpace V5.7 software to cluster the keyword co-occurrence knowledge graph to generate an automatic clustering label view, as shown in Fig. 2. The clustering module value $\mathrm{Q}$ is $0.8488(\mathrm{Q}>0.3$ is considered as significant clustering), indicating that the clustering effect is relatively significant. Clustering mainly focused on influencing factors, educational scientific research, dimensions, CiteSpace, policy implications, etc. 


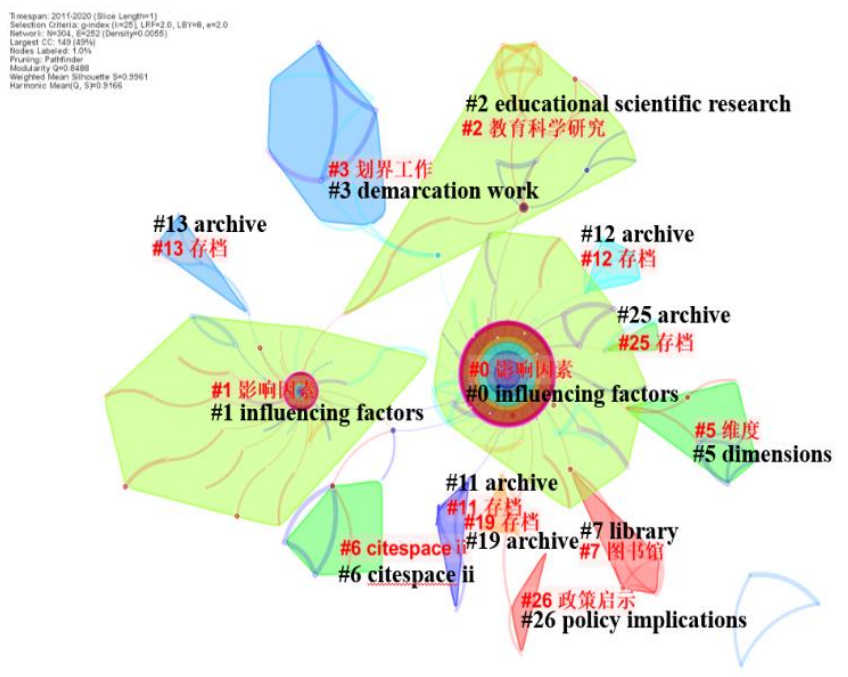

Note: The original figure is in Chinese; however, the English is translated.

Figure 2. Keyword knowledge graph in grounded theory literature

\subsection{Analysis of research priorities}

Among the high-frequency keywords, grounded theory, influencing factors and qualitative research have high intermediary centrality, which shows that they play an important role in mediating. It also plays a more significant role in the clustering map. Through the specific analysis of high-frequency keywords with relatively high intermediary centrality and clustering, the research focus can be further analyzed.

\subsubsection{Grounded theory and local management}

In this study, the search topic is grounded theory, and thus it is the most prominent keyword, appeared 335 times. Coding technology is a method and procedure to construct a domain theory based on the grounded theory paradigm. According to the recognition theory derived from data, there are obvious differences in coding principles and procedures followed by classical grounded theory, procedural grounded theory and constructive grounded theory. However, the three schools of grounded theory all follow the epistemological principle that theory originates from practice and practice tests theory. This is the core idea and common spirit of all schools of grounded theory, and also the key point that grounded theory can make contributions to the future local management research in China. Therefore, Xu Shuying et al. believed that "it is essential to apply the grounded theory research method to study Chinese and foreign enterprises under the Chinese background in order to accurately grasp the complex
Chinese background and to obtain models with high internal and external validity" [6].

\subsubsection{Influencing factors and qualitative research}

Grounded theory is produced in the field of sociology. It aims to obtain and analyze data in a systematic way to construct a theory in line with the actual situation, and to guide practical activities while explaining practical problems. Therefore, the influencing factors are also very prominent, with a frequency of 50 times.

Grounded theory is a methodology to discover theories from materials and data, which is closely related to social practice. Therefore, qualitative research is also prominent, with 14 times of frequency. Qualitative research method is one of the basic methods of organizational management research, and it is also one of the important research methods for the establishment of management theory. It is a research strategy, and its purpose is to understand the dynamic process in a single situation. Qualitative research is more suitable to explore the evolution process and reasons under a certain situation. It is suitable for understanding phenomena, exploring new concepts and ideas, and even creating new theories. With regard to principles, steps and methods, qualitative research methods have reached a consensus. Compared with the empirical research method, the qualitative research method is helpful to construct the Asian native theory, which is very consistent with the Chinese culture.

\subsection{Analysis of development context}

Top 9 Keywords with the Strongest Citation Bursts



Figure 3. Emergent words in grounded theory literature

CiteSpace software has the function of keyword cluster analysis, and provides sudden detection technology. It can detect words with high frequency change rate (i.e., burst terms) from a large number of keywords, and select the professional terms with sudden increase of frequency, which is particularly suitable for characterizing the current research frontier. Fig. 3 lists 9 salient keywords in the order of the emergence years of salient values. It can be found that industrial clusters, business models, supply chains and 
enterprise clusters were more prominent from 2011 to 2014 , indicating that scholars began to use grounded theory to explore the correlation between influencing factors and variables in the economic field at this time. Since 2014, the sports industry has continued to be prominent. This is inseparable from the fact that the State Council made national fitness a national strategy in 2014 by issuing "several opinions on accelerating the development of sports industry and promoting sports consumption".

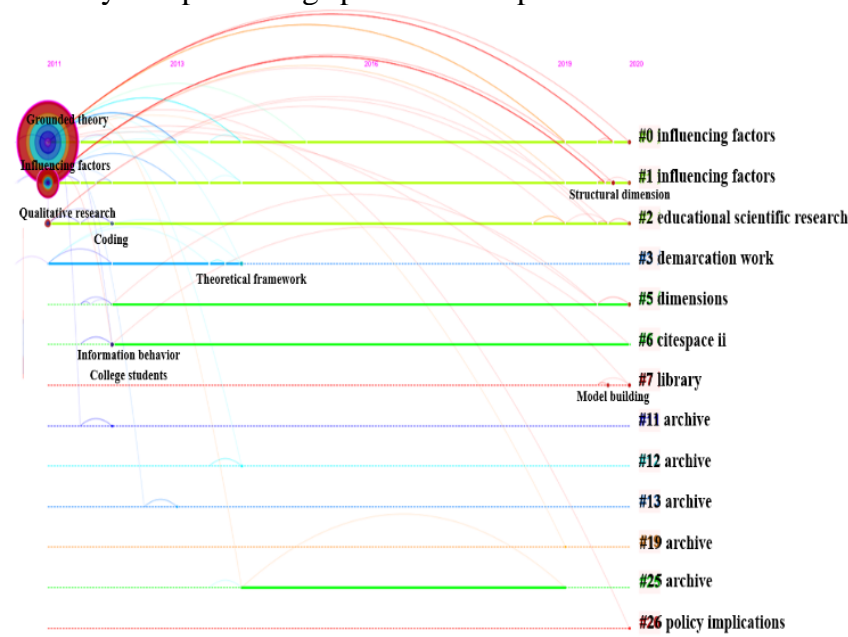

Figure 4. Timeline view of keyword clustering in grounded theory literature

In order to more vividly show the development trend of literature research on grounded theory from 2011 to 2020, this study uses CiteSpace V5.7 software to draw the timeline view of keyword clustering (as shown in Fig. 4). Through this graph, the keywords contained in each cluster can be transformed and associated with other keywords. It can be seen that the grounded theory has been applied to the research of influencing factors from the very beginning, and has been a great achievement in the field of educational science research. Grounded theory is conducive to empowering China's educational research and seeing more local characteristics of research phenomenon. At present, China's educational research is seriously "colonized" by western "grand theory". Most researchers are using western conceptual framework and lack of their own local theoretical perspective. By using grounded theory to return to China's educational practice and refining local theories in the face of empirical data, it may be able to obtain research results closer to China's educational reality.

\section{DISCUSSION}

In the face of the difficulties and challenges in reality, Han Wei has realized that "the research on Chinese management issues avoids the complexity of Chinese situations, lacks the relevance to explain Chinese problems, and mostly extends and mends Western orthodoxy" [1]. Xu Shuying et al. also repeatedly pointed out that "we ignore the correlation between theory and practice, and we forget that the ultimate goal of science is to improve human living conditions, and we also forget that the significance of management theory is to improve management practice" [6]. Management is a discipline in which learning is applied. The source and essence of management science have determined that it must also be a practice-oriented discipline, and practice is the source and foundation for the survival and development of this discipline.

For the current management circles in China, it is of great significance to use grounded theory to explore the law behind the local phenomenon of Chinese management and construct the theory for the improvement of Chinese management theory and practice level, as well as the prosperity of the country. At present, Chinese enterprises are in a period of dramatic changes in business environment, numerous management problems and serious lack of theoretical guidance. On the one hand, Chinese enterprises, especially the small and medium-sized private enterprises, have paid a high "tuition" and huge price in practice under the guidance of the western management theory. However, their management level has not been substantially improved. On the other hand, the experience of some successful enterprises also needs to be summarized and promoted, thus benefiting more enterprises and promoting the overall improvement of Chinese enterprises' management level [2].

\section{CONCLUSION}

Because the grounded theory cannot adopt the same rigorous data processing and analysis process as empirical research, the grounded theory has a late start in China. However, qualitative research has been paid increasingly attention in the field of domestic management research in recent ten years. Grounded theory research has also been gradually rising in China, and scholars have higher recognition of grounded theory.

The research results show that grounded theory is widely used in management, education, economics, physical education, etc. In recent ten years, the literature research content mainly centers on the influencing factors, dimension exploration and theory construction under various themes. "Grounded spirit" is the inherent requirement and essential embodiment of the management discipline. Currently, domestic management urgently needs the academic circles to actively participate in the fertile soil of Chinese local management practice. It is hoped that scholars can use grounded theory to refine Chinese local management theory, return to Chinese practice to obtain research results closer to Chinese reality, which can be used to guide future practice.

\section{REFERENCES}

[1] Han Wei. Discussion on Several Key Issues in the Construction of Local Chemistry Discipline [J]. Acta 
Management Sinica, 2009, 6 (6): 711-717. DOI:10.3969/j.issn.1672-884X.2009.06.001.

[2] Jia Xudong. A Preliminary Study on the Construction Paradigm of Chinese Local Management Theory Based on "Grounded Spirit" [J]. Journal of Management, 2016, 13 (3): 336-346.

DOI:10.14134/j.cnki.cn33-1336/f.2017.11.003.

[3] Zhang Jialiang, Liu Jun. Construction, Inheritance and Development of Management Theory: A

Qualitative Review from Western Classic Works and Papers [J]. Business Economy and Management, 2017 (11): 26-39. DOI:10.14134/j.cnki.cn331336/f.2017.11.003.
[4] Charmaz, K. C. "Constructing Grounded Theory: A Practical Guide Through Qualitative Analysis". International Journal of Qualitative Studies on Health and Well-Being, 1.3 (2006). DOI:10.3402/qhw.v1i3.4932.

[5] Chen Xiangming. Exploration on the Application of Grounded Theory in China's Education Research [J]. Peking University Education Review, 13.01 (2015): 215+188. DOI:10.19355/j.cnki.1671-9468.2015.01.002.

[6] Xu Shuying, Lv Li. Theoretical and Practical Issues of Chinese Local Management Research: An Interview with Xu Shuying. Journal of Management, 12.03 (2015): 313-321. DOI:CNKI:SUN: GLXB.0.2015.03.002. 